\title{
Ciencias Aeronáuticas: Nuevo campo académico en la UNAH
}

María Cristina Pineda de Carías

\section{Resumen}

Este artículo describe en términos generales los campos y actividades principales que comprenden las Ciencias Aeronáuticas. Se hace la distinción entre la aeronáutica militar y la aeronáutica civil, posicionando a esta última, en el marco legal nacional e internacional y de diferentes operaciones que se desarrollan a nivel mundial. Se presenta la oferta académica de la Universidad Nacional Autónoma de Honduras por medio de la Facultad de Ciencias Espaciales, y los retos para el establecimiento de este nuevo campo académico desde la Escuela de Ciencias Aeronáuticas.

Palabras claves: Ciencias Aeronáuticas, Aeronáutica Civil, Honduras, oferta académica universitaria.

\section{Abstract}

This article describes in general terms the fields and main activities that include Aeronautical Sciences. The distinction between the military aircraft and the civil aeronáutics is done by positioning the latter, in national and international legal framework and different operations carried out around the world. The academic offer of the National Autonomous University of Honduras by the Space Science Faculty, and the challanges to the establishment of this new academic field from the School of Aeronautical Sciences are presented.

Keywords: Aeronatical Sciences, Civil Aeronautics, Honduras, university academic offer

María Cristina Pineda de Carías, (mcpinedacarias@gmail.com), Decana Facultad de Ciencias Espaciales, Universidad Nacional Autónoma de Honduras - UNAH 


\section{Introducción}

Las Ciencias Aeronáuticas son un campo académico que, en la Nomenclatura Internacional de la UNESCO para los campos de Ciencia y Tecnología, cae dentro de las Ciencias Tecnológicas (Código: 33), y dentro de éstas, en las disciplinas de la ingeniería y tecnologías aeronáuticas (Código: 3301).

Surgen por la creación de un conjunto de conocimientos científicos y tecnológicos ligados a satisfacer la necesidad humana de querer volar, elevarse como los objetos que levanta el viento, o transportarse como lo hacen las aves de forma autónoma para ir de un lugar a otro, o de la necesidad trascendente de ir más allá del lugar donde estamos parados, surcar el cielo para comunicarnos, y para conocer el espacio profundo.

Para explicar de manera general el campo de las Ciencias Aeronáuticas, haré un breve repaso de algunos de los aspectos que comprende. Comenzaré por los aviones, esas magníficas máquinas voladoras que, desde los tiempos que los hermanos Wright lograron construir y poner uno en vuelo, han evolucionado hasta conseguir en nuestros días y en un futuro cercano, logros espectaculares.

Aquí me detengo para distinguir entre, lo que es construir o fabricar un avión o una aeronave; de otra cosa que es, comandar, dirigir o pilotear una aeronave. Del desarrollo de estas actividades han surgido, de una parte la industria de la fabricación de aeronaves; y de la otra, los pilotos, hombres y mujeres formados y capacitados para realizar con éxito los diferentes tipos de vuelo, a quienes se les suman los ingenieros y mecánicos de las aeronaves cuyo reto abarca también, realizar nuevos desarrollos, nuevos prototipos para cumplir objetivos cada vez más avanzados.

Disponer de aeronaves implica varias cosas. Implica, tener un lugar seguro donde guardarlas cuando no estén en uso. Un lugar que además, haya sido construido y equipado con requerimientos apropiados y condiciones especiales, desde donde se embarque una tripulación o carga para transportarse por el aire, hasta llegar a otro lugar, donde aterrizar y donde descargar la tripulación y la carga. Hablo de las instalaciones especializadas, que cumplen la función de ser el lugar de origen y el lugar de destino de un vuelo. De los aeródromos o aeropuertos, dotados de terminales, puestos de control, y pistas para el despegue y aterrizaje de los aviones. 
Dentro de los aeropuertos, obligado resulta disponer de una torre con amplia visibilidad y equipo de comunicación e información para controlar el tráfico aéreo de los alrededores, informar a los pilotos de las condiciones del tiempo y la meteorología, y del momento preciso para hacer el despegue o el aterrizaje. Estos lugares son las torres de control del tráfico aéreo.

\section{Actividades aeronáuticas}

En las sociedades organizadas se distinguen dos grandes campos para el desarrollo de las actividades derivadas de las ciencias aeronáuticas. La aeronáutica militar y a la aeronáutica civil.

La aeronáutica militar, tiene que ver con la seguridad y la defensa nacional. En Honduras, corresponde a la Fuerza Aérea Hondureña. De allí la necesidad de que Honduras forme a sus propios profesionales militares, tenga bases aéreas con aeropuertos y torres de control, flotas de aviones militares y talleres para su mantenimiento y reparaciones, y un conjuntos de regulaciones y disposiciones para poder operar bajo un régimen de leyes aplicables en casos emergencia, guerras 0 la defensa del país.

La aviación civil, tiene que ver con el transporte comercial de personas, mercancías y otros asuntos civiles o humanitarios, en un traslado desde un lugar de origen hasta otro de destino. Aquí hay que hacer referencia a las compañías aéreas, nacionales 0 extranjeras, que para satisfacer las demandas del transporte aéreo tienen flotas de aviones, y dentro de los aeropuertos sus centros de despacho de vuelo o de recibo de los pasajeros, que cuentan con permisos para operar en determinados aeropuertos, y en sus vuelos pueden atravesar rutas o carreteras aéreas dentro de los países, o rutas aéreas que cruzan ciudades y atraviesan países, o a veces los océanos, para enlazar lugares de origen y destino en cualquier lugar de la Tierra.

En todo lo concerniente a las actividades aeronáuticas, a medida que estas se han desarrollado, en una necesidad de los países se convirtió la supervisión de parte de los poderes públicos, que han tenido que regular el campo. Por ejemplo, en la Constitución de la República de Honduras se establece que el Estado, ejerce soberanía, jurisdicción completa y exclusiva sobre el espacio aéreo, cuyo dominio es inalienable e imprescriptible, sin desconocer los derechos legítimos similares 
con otros Estados, sobre la base de reciprocidad, ni afectar los derechos de libre navegación de todas las naciones conforme al Derecho Internacional en cumplimiento de los Tratados o Convenios ratificados por el Estado. La Ley de Aeronáutica Civil que es de orden público establece las normas que regulan la Aviación Civil en el territorio y espacio aéreo nacional. La Dirección General de Aeronáutica Civil (DGAC) es el ente regulador.

Honduras como Estado ha adoptado la política aérea de los Cielos Abiertos y de acuerdo a ello, otorga irrestrictamente a todos los países, con respecto a los servicios aéreos comerciales internacionales, regulares y no regulares, las cinco Libertades del Aire, limitadas únicamente por los aspectos de seguridad y responsabilidad señalados en la Ley. Estas cinco libertades son: 1) El derecho de sobrevolar el territorio nacional sin aterrizar; 2) El derecho de aterrizar en el territorio nacional por motivos técnicos pero no comerciales; 3) El derecho de desembarcar pasajeros, correo y mercancías procedentes del Estado de nacionalidad de la aeronave en el territorio nacional; 4) El derecho de embarcar pasajeros, correo y mercancías en territorio nacional con destino al Estado de nacionalidad de la aeronave; y, 5) El derecho concedido al Estado de nacionalidad de la aeronave de embarcar y desembarcar pasajeros, correo y mercancías procedentes y con destino a un tercer Estado.

En 1944, en la ciudad de Chicago, Estados Unidos, los Gobiernos de diferentes países del Planeta firmamos el Convenio sobre Aviación Civil Internacional, que hoy es reconocido por la Organización de Naciones Unidas. En su Preámbulo el Convenio establece: "Que el desarrollo futuro de la aviación civil internacional puede contribuir poderosamente a crear y a preservar la amistad y el entendimiento entre las naciones y los pueblos del mundo, mientras que el abuso de la misma puede llegar a constituir una amenaza a la seguridad general. Que es deseable evitar toda disensión entre las naciones y los pueblos y promover entre ellos la cooperación de que depende la paz del mundo.

El Convenio se divide en cuatro partes que tratan sobre: la Navegación Aérea, la Organización de la Aviación Civil Internacional, el Transporte Aéreo Internacional, y las Disposiciones Finales. Los 19 Anexos de este Convenio, son los que actualmente regulación la Aviación Civil Internacional. Regulan: 1) Licencias al personal; 2) Reglamento del aire; 3) Servicio Meteorológico para la navegación aérea internacional; 4) Cartas aeronáuticas; 5) Unidades de Medida; 6) Operación de Aeronaves; 7) Marcas de nacionalidad y de Matrícula de las aeronaves; 8) Aeronavegabilidad; 9) Facilitación; 10) Telecomunicaciones aeronáuticas; 11) Servicios 
de tránsito aéreo; 12) Búsqueda y salvamento; 13) Investigación de accidentes e incidentes de aviación; 14) Aeródromos; 15) Servicios de información aeronáutica; 16) Protección al medio ambiente; 17) Seguridad: Protección de la aviación civil internacional contra los actos de interferencia ilícita; 18) Transporte sin riesgos de mercancías peligrosas por vía aérea; 19) Gestión de la seguridad operacional. Como un organismo especializado de las Naciones Unidas existe la Organización de Aviación Civil Internacional (OACl). Sus objetivos estratégicos son la seguridad operacional, la seguridad de la aviación y la protección del medio ambiente y el desarrollo sostenible del transporte aéreo.

\section{Los profesionales de la aeronáutica civil}

Las actividades de la aeronáutica civil mencionadas, demandan también de profesionales con formaciones y competencias claramente establecidas en los ambientes laborales, para desempeñar puestos precisamente definidos. Entre ellos se pueden mencionar a los pilotos, el personal de cabina, los controladores de tránsito aéreo, los empleados de servicio al cliente, los manipuladores de equipaje, agentes de seguridad, y el personal de diseño, construcción y mantenimiento de aeronaves.

De acuerdo a mediciones y pronósticos globales y regionales de la $\mathrm{OACl}$, la distribución geográfica de aviones, entre los años 2010 y 2030 reporta: a) En Norte América: una disminución del $46 \%$ al 32\%; b) En Latinoamérica: más bien un incremento del $10 \%$ al $12 \%$; c) En África: subirá del $5 \%$ al $6 \%$; d) En Europa: subirá del 24\% al 26\%; e) En Medio Oriente: permanecerá estable en 3\%; f) En Asia: se incrementará del $12 \%$ al $21 \%$.

De acuerdo con la misma fuente, esto representará un crecimiento anual promedio del tráfico aéreo de: $6.27 \%$ en Asia; $5.8 \%$ en America Latina; $5.2 \%$ en Medio Oriente; $4.7 \%$ en África; $4.1 \%$ en Europa; 3.03\%: en Norte América. Por profesiones, en poblaciones globales proyectadas del 2011al 2030 representan crecimientos: de 463,388 a 980,799 pilotos; de 580,926 a 1,164,969 personal de mantenimiento; y de 87,024 a 139,796 controladores de tráfico aéreo, haciéndose evidente la necesidad de instrucción y formación en estos campos.

La seguridad del sistema de transporte aéreo mundial es por ahora el objetivo estratégico rector y fundamental de la Organización de Aviación Civil Interna- 
cional (OACI). Una evolución hacia una estrategia de la seguridad operacional de la aviación basada en el riesgo, se ha planteado.

Sin embargo, en los asuntos de seguridad de la aviación mundial, Honduras actualmente no se encuentra entre los Estados con implantación efectiva superior al promedio mundial. Por ello, para garantizar que la mejora continua de la seguridad operacional y la modernización armonizada de la navegación aérea mundial avancen en conjunto, es esencial la planificación de la seguridad operacional de la aviación a nivel mundial, regional y estatal. Es urgente pues, que profesionales nacionales de la aviación civil atiendan este asunto.

\section{La oferta académica de la UNAH}

Para responder a la demanda de formación de profesionales de las Ciencias Aeronáuticas en Honduras, particularmente de la Aeronáutica Civil, la Universidad Nacional Autónoma de Honduras, por medio de la Facultad de Ciencias Espaciales ha organizado el Departamento de Ciencias Aeronáuticas, que desde 2012 desarrolla actividades académicas de: docencia, investigación y vinculación de la Universidad con la Sociedad.

En materia de formación en Aeronáutica Civil, el Departamento de Ciencias Aeronáuticas ha trabajado en la Educación No Formal y en la Educación Formal.

En la Educación No Formal, elaboró la propuesta de un Diplomado en Gestión de Sistemas Aeroportuarios, la cual fue aprobada por la Vicerrectoría Académica para comenzar en 2013. A la fecha, una Primera Edición de este Diplomado se completó por 31 profesionales del campo aeronáutico nacional, realizando las actividades principalmente en el Aeropuerto Internacional de Toncontín de Tegucigalpa. Una segunda edición de este diplomado se ha programado para ser desarrollada en la UNAH Valle de Sula, para beneficio de la región norte del país, aprovechando las instalaciones del Aeropuerto Internacional Villeda Morales de San Pedro Sula.

En materia de Educación Formal, el Departamento de Ciencias Aeronáuticas ha trabajado en el Diagnóstico y Propuesta de Planes de Estudio de Carreras Cortas de Técnico Universitario y de Licenciatura en Ciencias Aeronáuticas. Las orientaciones en ambas carreras son de Piloto de Ala Fija, Piloto de Ala Rotatoria y de Despachador de Vuelo. Las propuestas ya han entrado al proceso de aprobación, encontrándose actualmente en la etapa de dictámenes de diferentes instan- 
cias universitarias, previo a su aprobación definitiva por parte del Consejo de Educación Superior. En 2015 se espera que inicie el funcionamiento de estas carreras.

\section{Los retos}

En el año de 1960, nació en Honduras la Escuela de Aviación Civil Toncontín, la cual graduó a varias promociones de pilotos. Como una actividad de Vinculación Universidad Sociedad reciente del Departamento de Ciencias Aeronáuticas y la Fundación del Museo del Aire se hizo un Homenaje a las Pioneras de la Aviación en Honduras. Una de ellas era doña Lesbia Ponce de Madrid, quien dirigió la Escuela de Aviación Toncontín. La otra, María Elena Mondragón de Torres, primera mujer aviadora de Honduras. En esta oportunidad doña Lesbia de Madrid comentó: "Con el paso del tiempo observé que en las líneas aéreas comerciales trabajaban pilotos en su mayoría extranjeros y algunos pocos egresados y retirados de la Fuerza Aérea Hondureña". Hoy la situación no ha cambiado mucho, y por eso el reto es mayor.

En la Facultad de Ciencias Espaciales, como parte de su Plan Estratégico para el Período 2014-2018 se han planteado dos grandes retos:

- El Primero: La Consolidación de la Estructura Académica Organizativa de la Facultad, que en el campo de las Ciencias Aeronáuticas comprende la creación de la Escuela de Ciencias Aeronáuticas, con los departamentos académicos de Aeronáutica Civil y Aviación.

- El Segundo: El Desarrollo e Innovación Curricular fortalecido por la Investigación y la Vinculación con la Sociedad. Para ello hemos planteado en la Educación No Formal: continuar con la oferta de varios Diplomados; en la Educación Formal, el desarrollo de Carreras de Técnicos Universitarios y Licenciaturas en Ciencias Aeronáuticas. Para el relevo docente, esperamos desarrollar una Maestría en Sistemas Aeroportuarios con el apoyo de la cooperación internacional.

La oferta académica en el campo de las Ciencias Aeronáuticas, está abierta en la Facultad de Ciencias Espaciales de la Universidad Nacional Autónoma de Honduras. Para desarrollarla, esperamos contar con el apoyo institucional, y de organizaciones públicas y privadas vinculadas a la Aeronáutica Civil nacional, regional e internacional. 


\section{Agradecimiento:}

Se agradece a la Universidad Nacional Autónoma de Honduras en el Valle de Sula (UNAH-VS), para que la Decana de la Facultad de Ciencias Espaciales, con el apoyo del personal docente del Departamento de Ciencias Aeronáuticas, presentara esta conferencia en el marco del II Congreso de Investigación: "La Investigación Científica en la Sociedad del Conocimiento".

San Pedro Sula, 7 de octubre de 2014. 